\title{
MEASUREMENTS OF FIELD DECAY AND SNAPBACK EFFECT ON TEVATRON DIPOLE AND QUADRUPOLE MAGNETS *
}

\author{
G.V. Velev ${ }^{\dagger}$, G. Ambrosio, G. Annala, P.Bauer, R. Carcagno, J.DiMarco, H. Glass, R. Hanft, R. \\ Kephart, M.Lamm, M. Martens, P.Schlabach, C. Sylvester, M. Tartaglia, J. Tompkins \\ FNAL, Batavia, IL 60510, USA
}

\begin{abstract}
Since the beginning of 2002 an intensive measurement program has been performed at the Fermilab Magnet Test Facility (MTF) to understand dynamic effects in Tevatron magnets. Based on the results of this program a new correction algorithm was proposed to compensate for the decay of the sextupole field during the dwell at injection and for the subsequent field "snapback" during the first few seconds of the energy ramp. Beam studies showed that the new correction algorithm works better than the original one, and improves the Tevatron efficiency by at least 3\%. The beam studies also indicated insufficient correction during the first $6 \mathrm{~s}$ of the injection plateau where an unexpected discrepancy of 0.15 sextupole units of extra drift was observed. This paper reports on the most recent measurements of the Tevatron dipoles field at the beginning of the injection plateau. Results on the field decay and snapback in the Tevatron quadrupoles are also presented.
\end{abstract}

\section{INTRODUCTION}

Field errors from persistent currents play a significant role in the complex operation of modern superconducting accelerators due to their large amplitude and time dependence. For example the effects from normal sextupole $\left(b_{2}\right)$ field errors, observed during the injection plateau at the early stage of the Tevatron operation, were unacceptable and corresponding time dependent corrections were invented [1].

For the ongoing Run II, the Tevatron normal sextupole compensation during injection went through several stages of improvement. Recently a new set of sextupole corrections was obtained from a systematic series of magnetic measurements performed at MTF. A new algorithm to control the Tevatron normal sextupole $\left(b_{2}\right)$ during injection was put in place. The result from magnetic measurements was distilled into five proposals for optimization of the Tevatron operation as follows: 1) remove the precycle(s) after the end of a successful store; 2) use a constant duration for the back-porch (the current profiles, back-porch, flattop and injection porch definitions used in our measurements are described in detail in ref.[2]); 3) use only one precycle with an increased length of $\sim 60 \mathrm{~min}$. if the store ended abnormally; 4) adopt the gaussian form (3) from ref. [2] for the snap-back compensation and 5) use the logarithmic form

\footnotetext{
*Work supported by the U.S. Department of Energy †velev@,fnal.gov
}

$$
\Delta b_{2}=b_{2,1} \cdot \log \left(\frac{t+t_{s}}{t_{s}}\right) .
$$

for the decay correction during the injection plateau defined in [2]. These proposals allowed a reduction in the number of parameters in the correction algorithms during injection and they increased the reproducibility between accelerator stores.

As a result of implementation of the proposal the Tevatron luminosity was increased by at least 3\%. In details these proposals were discussed in the papers $[2,3]$ presented at EPAC 2004.

This paper is a follow-up to previous publications $[2,4]$ presented at PAC2003 and EPAC2004 conferences. The first part of the paper presents our measurements on the Tevatron dipoles with very short injection plateaus typically smaller than $30 \mathrm{~s}$. In the second part of the paper results on the field decay in the Tevatron quadrupoles are also summarized.

\section{SEXTUPOLE FIELD DECAY IN TEVATRON DIPOLES AFTER A SHORT INJECTION PORCH}

The $b_{2}$ corrections were tested and optimized using beam based studies. A beam study was performed with a very short injection porch $(\sim 6 \mathrm{~s})$ to measure the chromaticity on the Tevatron ramp in a situation where the injection porch drift and snapback are minimal [5]. These measurements indicated a discrepancy on the level of 0.13-0.20 units (the field multipoles are reported at $25.4 \mathrm{~mm}$ reference radius) between the average Tevatron $\mathrm{b}_{2}$ component and the correction algorithm described by equation (1) (see Fig.2 in ref. [5]). The discrepancy vanishes approximately after the first 60 second of the beginning of the injection plateau.

The $6 \mathrm{~s}$ injection porch condition was not explored in the previous magnet test campaign, in which the shortest injection porch duration was set to $60 \mathrm{~s}$. A new systematic series of measurements with short duration injection porches $(6,12,20$ and 30s) were carried out. Furthermore, a ramp profile was executed which was as close as possible to the Tevatron beam study profile, where the discrepancy was observed (10 s flattop, $6 \mathrm{~s}$ back-porch and 6 injection porch). Four Tevatron dipoles (TB0834, TC0604, TC1130, TB1136) from the spares-pool were measured.

A typical $b_{2}$ hysteresis loop from the Tevatron beamtype study profile with 6 s injection porch is shown in Fig. 1. A standard procedure, described in ref. [2,4], is used 


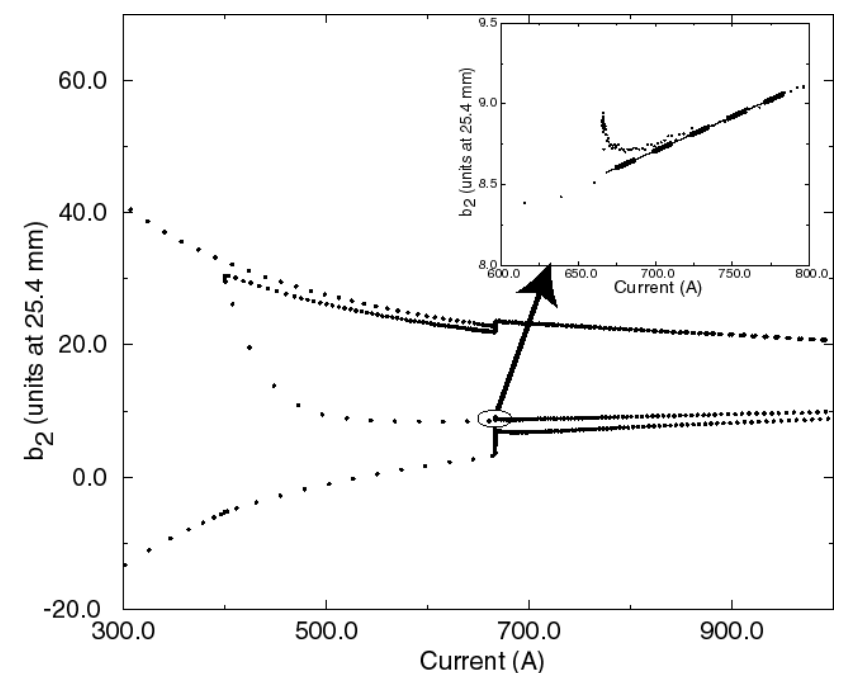

Figure 1: Typical $b_{2}$ hysteresis loop with short injection porch. The upper insert shows the snap-back after $6 \mathrm{~s}$ of injection porch and the line approximation of the loop in the snap-back's region

to decouple the decay and snapback from the underlying loop. In short, the $b_{2}$ values were linearly parametrized as a function of the current in the region of $0.7-0.78 \mathrm{kA}$, extrapolated to the injection plateau at $0.66 \mathrm{kA}$ and subtracted from the sextupole loop at $0.66 \mathrm{kA}$ (the upper insert in Fig.1). An unexpected fast decay of approximately 0.2 units and accompanying snapback is observed. In general the logarithmic form (1) can be used to estimate the drift amplitude $\Delta \mathrm{b}_{2}$ after 6 s on the injection porch. For the time constant $t_{s}=170 \mathrm{~s}$, determined from beam measurements with a flattop longer than $60 \mathrm{~min}$ and 5 min back-porch conditions, formula (1) predicts 0.017 units which is $\sim 10$ times smaller than the observed effect.

To achieve direct comparison with (1) we performed measurements with current profiles which corresponds to $\mathrm{t}_{\mathrm{s}}=170 \mathrm{~s}$ and with a short duration of the injection porch $(6,12,20$ and $30 \mathrm{~s})$. Fig. 2 shows the snapback curves for the magnet TC0604. Similar decay amplitudes and snapback times were obtained for magnet TB0834. The results are consistent with the observation a fast decay in first $6 \mathrm{~s}$ with amplitude of approximately 0.2 units, alike to what was observed in the Tevatron beam measurements.

The third measured magnet, TC1130, showed a large deviation from the pattern. The decay and snapback for this magnet is presented in the insert of Fig. 2. One observes that the decay amplitude for this magnet is much larger, on the order of 1 unit. One hypothesis is that TC1130 was produced from a different cable batch than the other two magnets. A fourth magnet, TB1136 close in production time to $\mathrm{TC} 1130$, was examined. It performed like the first two magnets with decay amplitude of 0.25 units after first $6 \mathrm{~s}$ of the injection porch.

The decay processes were occurring for relatively short period of time, which initially pointed to a direction of a possible connection to the ramp eddy currents in these magnets. Two of the dipoles (TC0604 and TC1130) were extensively tested for eddy currents, measuring the hysteresis loop width dependence on different ramp rates.

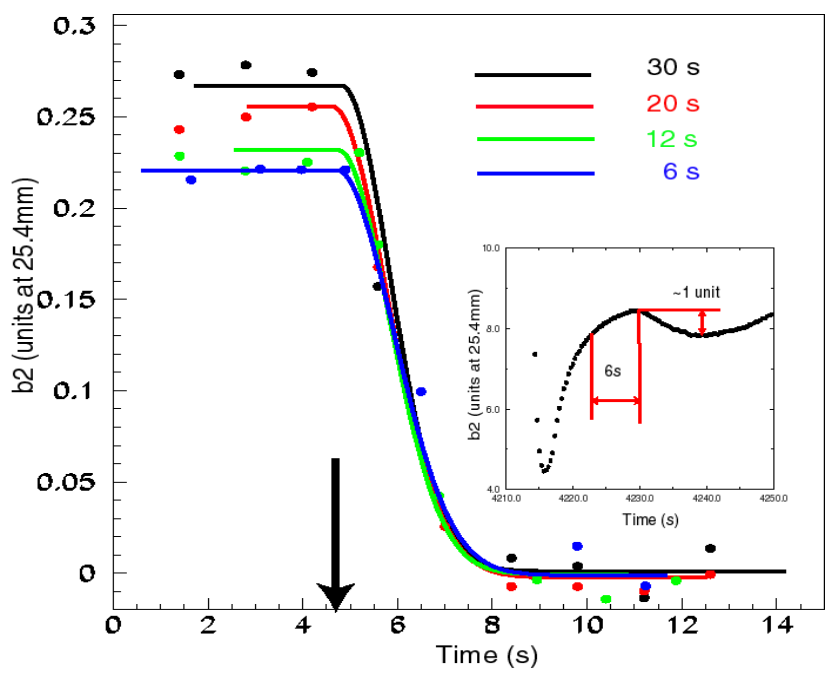

Figure 2: Snapback of the sextupole component in TC0604 for a plateau at injection of 6,12,20 and $30 \mathrm{~s}$ for 60 min flattop and 5 min back-porch. A fast decay of approximately 0.2 units is observed after first $6 \mathrm{~s}$. The arrow shows the beginning of the snapback. The insert shows the decay and snapback for TC1130 in case of $6 \mathrm{~s}$ injection porch

It was found that a change the current ramp rate from 10 to $80 \mathrm{~A} / \mathrm{s}$ resulted an increase of the $b_{2}$ hysteresis loop width by $0.1 \pm 0.05$ units in TC0605 and by $3.6 \pm 0.06$ in TC1130. The preliminary conclusion is that eddy currents can be considered a possible source for the observed fast decay in the magnets. Additional testing is needed to confirm or reject this hypothesis.

In case of the Tevatron operation at injection an additional constant value of $b_{2}=0.13$ units was adjusted to normal sextupole correction algorithm for the first $6 \mathrm{~s}$ as described in [5].

\section{FIELD DECAY AT INJECTION PORCH IN TEVATRON QUADRUPOLES}

The results reported in ref. [2,4] and in the first part of this paper address the decay and snapback in Tevatron dipoles. The next major component of the Tevatron ring is the qudrupole magnets.

The Tevatron correction scheme includes magnetic corrector elements up to the octupole component of the fields. Therefore we have investigated decay and snapback in the main field, and possible dynamic effects in the sextupole and octupole components in Tevatron quadrupole magnets. In addition, to create a consistent picture, a result on dodecapole decay and snapback is also presented.

Four Tevatron qudrupoles from the spares pool, n9921f, tq214f, tq245f and tq103f were measured cold. All have the same coil design but differ in length: the tqtype is a standard 66 inches qudrupole magnet while n9921f has a length of 99 inches.

Fig. 3 shows the result for the typical decay in the main quadrupole field for magnet n9921f after a $30 \mathrm{~min}$ injection porch. The average decay amplitude of 


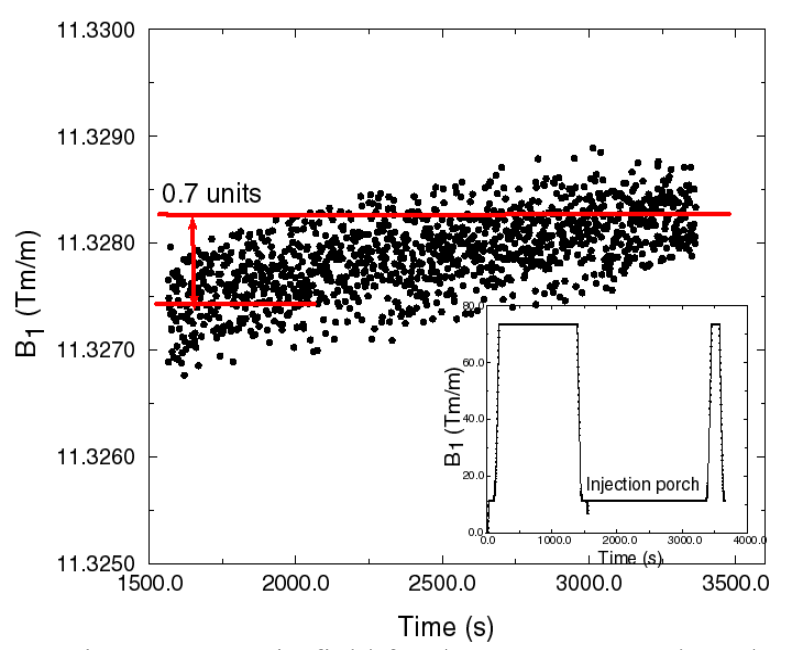

Figure 3: A main field for the Tevatron quadrupole N9921F after $30 \mathrm{~min}$ injection porch. The insert shows the main field dependence versus time for this measurement. A decay of 0.7 units was observed

$0.67 \pm 0.10$ units (Table 1) is somewhat smaller than that observed in the dipoles which we found to be on the order of 1 unit (preliminary result). This may generate a deviation in the optimal ratio of the integrated quadrupole to dipole fields and be a source of tune drift instability in Tevatron operation.

Table 1 summarizes the decay amplitudes for the quadrupole main field, and the normal sextupole and normal dodecapole components. A small effect was found in the normal sextupole in tq214f, but the overall value is consistent with zero. Furthermore no evidence was found for the decay in the octopole field as it was expected.

The normal dodecapole shows an expected decay with average amplitude of $0.34 \pm 0.07$ units (Table 1). The average snapback time, accordingly Eqn. (3) from ref. [2], was found to be $5.2 \pm 0.4 \mathrm{~s}$. The result is consistent with the mean value of $5.1 \mathrm{~s}$ obtained from Tevatron dipoles [2].

For one of the quadrupoles, tq245f, a measurement with a $60 \mathrm{~min}$ flattop, $5 \mathrm{~min}$ of back-porch and short duration ( 20 s) injection porch was executed. The analysis revealed small $b_{5}$ decay on the level of approximately 0.13 units. Additional testing is needed to create a consistent picture of possible fast decay in Tevatron quadrupoles.

\section{CONCLUSION}

For first time a fast decay and accompanying snapback was observed in the sextupole field component in the Tevatron dipoles. The measurements showed that an average decay of 0.25 units occurs after the first $6 \mathrm{~s}$ of the injection porch. This decay is not described with the long-term parametrization (Eqn. 1) typically used for the $\mathrm{b}_{2}$ operational corrections. Furthermore there is a hint that this fast decay may depend on eddy current effect in the cable. The results from these measurements may be useful for the future operation of the LHC, where the
Table 1: The decay amplitude in units for the quadrupole main field, normal sextupole and normal dodecapole for the four measured Tevatron quadrupoles after $20 \mathrm{~min}$ flattop, $1 \mathrm{~min}$ back-porch and $30 \mathrm{~min}$ injection porch.

\begin{tabular}{|c|c|c|c|}
\hline Quad & $\begin{array}{c}\text { Main Field } \\
\text { (units) }\end{array}$ & $\begin{array}{c}\mathrm{b}_{2} \\
\text { (units) }\end{array}$ & $\begin{array}{c}\mathrm{b}_{5} \\
\text { (units) }\end{array}$ \\
\hline n9921f & $0.7 \pm 0.2$ & $<0.1$ & $0.33 \pm 0.09$ \\
\hline tq214f & $0.9 \pm 0.3$ & $0.21 \pm 0.07$ & $0.31 \pm 0.08$ \\
\hline tq245f & $0.7 \pm 0.2$ & $0.13 \pm 0.10$ & $0.25 \pm 0.09$ \\
\hline tq103f & $0.4 \pm 0.2$ & $<0.1$ & $0.46 \pm 0.11$ \\
\hline Average & $0.67 \pm 0.1$ & $\sim 0.1 \pm 0.10$ & $0.34 \pm 0.07$ \\
\hline
\end{tabular}

injection porch is expected to be relatively short and accurate dynamic corrections are critical to the beam stability.

The main field, $b_{2} / a_{2}, b_{3} / a_{3}$ and $b_{6}$ possible decay and snap-back effects at injection were studied on four Tevatron quadrupoles. No decay (consistent with zero decay amplitude) was found in the low order unallowed normal and skew harmonics. In addition, it was confirmed that the dynamic effects in quadrupoles are consistent with those observed in the Tevatron dipoles.

\section{REFERENCES}

[1] D.A.Finley et al., "Time dependent chromaticity changes in the Tevatron", Proceeding of Part. Acc. Conf., Washington, DC, 1987 and D.E. Johnson and D.A. Herrup "Compensation of time Varying Field in the Tevatron Superconducting Magnets", Proceedings of PAC 1989.

[2] G. Velev et al., "Measurements of Sextupole Decay and Snapback in Tevatron Dipole Magnets", Proceedings of EPAC 2004.

[3] P.Bauer et al., "Proposals for Improvements of the Correction of Sextupole Dynamic Effects in the Tevatron Dipole Magnets", Proceedings of EPAC 2004.

[4] G. Velev et al., "Measurements of Field Decay and Snapback Effect on Tevatron Dipole Magnets", Proceedings of PAC 2003

[5] M. Martens et al., "Studies of the Chromaticity, Tune, and Coupling Drift in the Tevatron", MOPA010, These Proceedings. 